\title{
Dynamically induced effective interaction in periodically driven granular mixtures
}

\author{
Massimo Pica Ciamarra * Antonio Coniglio, and Mario Nicodemi \\ Dipartimento di Scienze Fisiche, Universitá di Napoli 'Federico II', CNR-Coherentia, INFN, 80126 Napoli, Italia I
}

(Dated: October 9, 2018)

\begin{abstract}
We discuss the microscopic origin of dynamical instabilities and segregation patterns discovered in granular mixtures under oscillating horizontal shear, by investigating, via molecular dynamics simulations, the effective interaction between like-particles. This turns out to be attractive at short distances and strongly anisotropic, with a longer range repulsive shoulder along the direction of oscillation. This features explain the system rich phenomenology, including segregation and stripe pattern formation. Finally, we show that a modified Cahn-Hilliard equation, taking into account the characteristics of the effective interaction, is capable of describing the dynamics of the mixture.
\end{abstract}

Dynamical instabilities and pattern formation, so important in Fluid Mechanics, have just begun to be discovered in granular materials [1, 2], which are collections of macroscopic particles interacting via dissipative forces where thermal effects are negligible. Understanding their origin in these systems, which is crucial for scientific reasons and substantial industrial applications, is today a challenge as granular systems cannot be simply described by usual Statistical or Fluid Mechanics 2, 3, 4, 5, 6, 7]. In this Letter we make a step further in this direction by showing that the study of peridocially driven binary granular mixture can be reduced to that of a thermal monodisperse undriven system of particles interacting via an effective potential. This result can be considered as an extension, to the non-thermal and driven case, of the 'depletion potential' approach introduced by Asakura and Oosawa [8] to map an undriven thermal binary mixture in a monodisperse system. However, while the depletion potential has a purely entropic origin as related to the size difference of the two components, the effective interaction we introduce here has a purely dynamical origin, as it results from the different response of the mixture components to the oscillating drive. Our approach could be also of value in the study of driven thermal binary mixutres..

We apply our ideas to a granular mixture subject to horizontal oscillations, a system previously investigated both experimentally and numerically [2, 9, 10, 11], whose complex and not well understood phenomenology encompasses both instabilities and segregation. First we determine, via Molecular Dynamics (MD) simulations, the effective interaction between like-particles of this mixutre when submitted to horizontal oscillations. Then we show that simulations of just one species of particles interacting via this effective interaction, in absence of horizontal oscillations, reproduces the phenomenology of the oscillated granular mixture. Finally, guided by the main properties of the effective interaction between like particles, we introduce a phenomenological Cahn-Hilliard equation which again reproduces the observed phenomenology, but also allows for analytical predictions.

Model - We have investigated via MD simulations a two-dimensional model [2] of the experiment of Ref. [9], where a monolayer of granular mixture is placed on a horizontally oscillating tray of size $160 D \times 40 D(D=$ $1 \mathrm{~cm})$. In our model, contacting particles interact via a spring dashpot repulsive force with constant coefficient of restitution $e=0.8$, and the interaction between a grain and the oscillating tray is given by $\mathbf{f}_{\text {tray }}=-\mu\left(\mathbf{v}-\mathbf{v}_{\text {tray }}\right)$, where $\mathbf{v}_{\text {tray }}(t)=2 \pi A \nu \sin (\nu t) \mathbf{x}$ is the velocity of the tray and $\mathbf{v}$ the velocity of the disk, plus a white noise force $\xi(t)$ with $\left\langle\xi(t) \xi\left(t^{\prime}\right)\right\rangle=2 \Gamma \delta\left(t-t^{\prime}\right)$ (see [2, 12] for details). The two components of our mixture have masses $m_{\mathrm{h}}=1$ $\mathrm{g}, m_{1}=0.03 \mathrm{~g}$, viscous coefficient $\mu_{\mathrm{h}}=0.28 \mathrm{~g} \mathrm{~s}^{-1}$ and $\mu_{1}=0.34 \mathrm{~g} \mathrm{~s}^{-1}$, the white noise has $\Gamma=0.2 \mathrm{~g}^{2} \mathrm{~cm}^{2} \mathrm{~s}^{-3}$, and the tray oscillates with amplitude $A=1.2 \mathrm{~cm}$ and frequency $\nu=12 \mathrm{~Hz}$. The diameters of the two species considered here are equal, $D_{h}=D_{l}=D=1 \mathrm{~cm}$, to remark that no "entropic" depletion forces are present.

Effective interaction - In order to determine the effective interaction we have performed simulations where two heavy disks are placed at fixed relative positions $\mathbf{r}_{12}=\mathbf{r}_{1}-\mathbf{r}_{2}=(x, y)$ in a system of lighter disks covering an area fraction $\phi \simeq 0.63$ (we have sampled the range $0<x, y<6 D$ ). When the tray oscillates horizontally the two heavy disks are moved as a single object of mass $M=2 m_{\mathrm{h}}$ subject to a force $\mathbf{f}=\mathbf{f}_{1}+\mathbf{f}_{2}+\left(2 \mathbf{f}_{\text {tray }}+2 \mathbf{f}_{\text {noise }}\right)$, where $\mathbf{f}_{1}\left(\mathbf{f}_{2}\right)$ is the force acting on particle 1 (2) due to collisions with lighter disks. The two disks can translate horizontally and vertically, but $\mathbf{r}_{12}$ remains fixed. The effective force that particle 1 exerts on particle 2 , averaged over one period of oscillation $T$, is given by

$$
\mathbf{f}^{\mathrm{eff}}=\left(f_{x}^{\mathrm{eff}}, f_{y}^{\mathrm{eff}}\right)=\frac{1}{2 T} \int_{0}^{T}\left[\mathbf{f}_{1}(t)-\mathbf{f}_{2}(t)\right] d t .
$$

In the study of different systems, as for instance for two spheres in a oscillating fluid flow, an analytical expression for the effective interaction force can be in principle found.

Our numerical results show that this force is attractive at short distances, and strongly asymmetrical in the $x y$-plane: it has a repulsive shoulder at long distances, which vanishes along the $y$ axis. This is shown in Fig. 11 where we plot the radial component of the effective interaction force along directions forming an angle $\theta$ with the $x$ axis. The effective force is weakly dependent on 


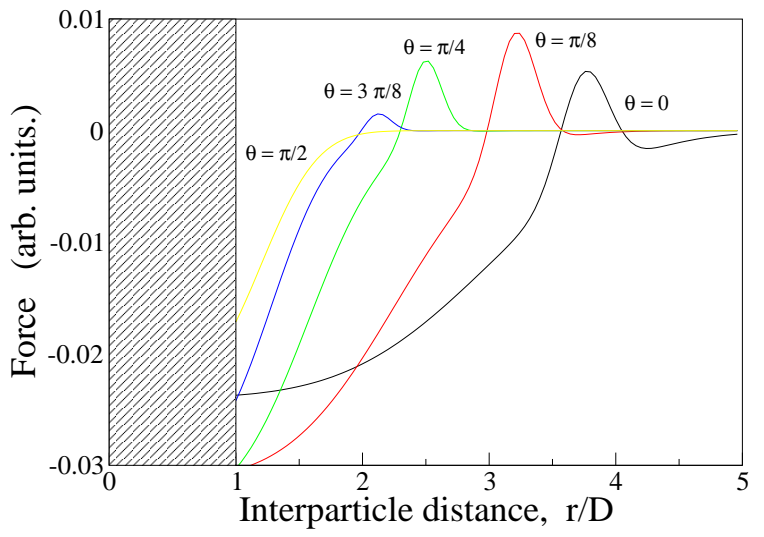

FIG. 1: (color on line) Radial component of the effective force along directions forming an angle $\theta$ with the $x$ axis. For $r / D<1$ the force is strongly (out of scale) repulsive due to steric constraints.

$\Gamma$ (in the investigated range $0<\Gamma<10 \mathrm{~g}^{2} \mathrm{~cm}^{2} \mathrm{~s}^{-3}$ ), and its amplitude increases with the area fraction of the smaller species. Variations of the amplitude and frequency of oscillation do not change the qualitative features of the effective force (asymmetry, attraction at short distances and repulsive shoulder), but they change the range of attraction and the position of the repulsive shoulder. Rigourously, the effective force $\mathbf{f}^{\text {eff }}$ cannot be derived by an effective scalar potential, as the curl of $\mathbf{f}^{\text {eff }}$ $\left(\nabla \times \mathbf{f}^{\mathrm{eff}}=\partial_{y} f_{x}^{\text {eff }}-\partial_{x} f_{y}^{\text {eff }}\right)$ varies in space (the presence of a solenoid component in the effective interaction has been also reported in 13] for a system of two disks in a constant fluid flow). However, as the solenoid component of $\mathbf{f}^{\text {eff }}$ appears to be negligibe with respect to its irrotational component [18], an effective interaction scalar potential can be introduced within a good approximation.

The effective force we derive (see Fig प) appears to be the key ingredient in understanding the phenomenology of our system. It follows from the different response of the two species of particles to the oscillating drive (the tray), which are forced to oscillate with different amplitues and phases due to their differences in mass and friction coefficient. A qualitatively understanding of the microscopic origin of the effective force can be obtained by considering the simpler case where two disks of a given species (striped disks in Fig. 2, a, b) are immersed in stream of disks of a different species flowing along the $x$ axis with velocity $v>0$. Fig. 22 illustrates that, as $\theta_{1}<\theta_{2}$, the majority of the collisions experienced by grain $\mathrm{A}$ push it closer to grain $\mathrm{B}$, explaining the attraction between particles $\mathrm{A}$ and $\mathrm{B}$ along $y$. Fig. 20 shows that, for small distances, particle A screens particle B, inducing an effective attraction between them along $x$. For larger distances, and in the presence of the oscillating drive, light disks tend to be caged between the two heavy ones, and their density may become higher than average, as shown
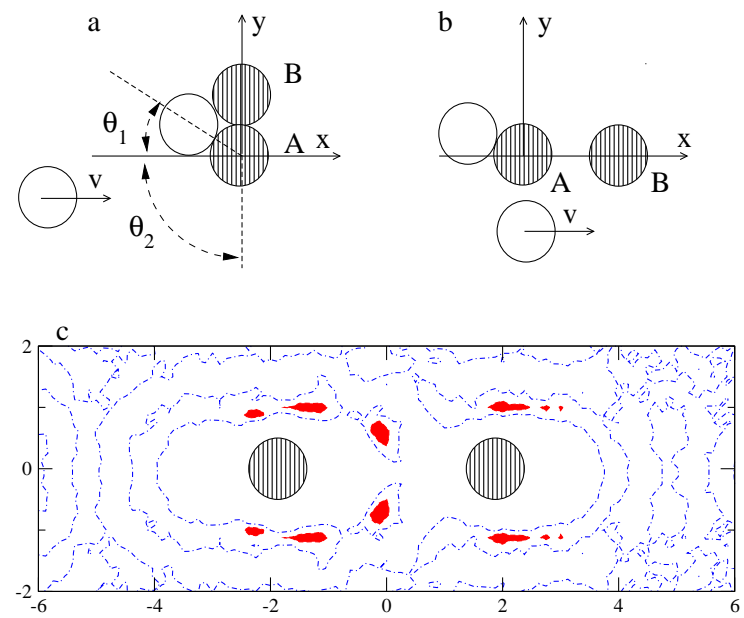

FIG. 2: (color on line) The effective interaction between two heavy disks is determined via screening effects by their relative position, as discussed in the text an exemplified in Panels a,b. Panel c is a contour level plot of the density field of light disks in an oscillating system. It shows that when the distance between the two heavy disks along the direction of oscillation increases light disks are caged between them giving rise to an effective repulsion (see text). Panel c has been obtained by recording the position of all particles after $1 / 4$ of oscillation in the frame of reference centered in the mid-points between the two heavy disks (kept at the fixed distance of $3.75 \mathrm{D}$ ), and averaging over 100 configurations. The regularity of the isodensity lines is due to steric constrains. Filled area represent regions of density above the average.

in Fig. 2r. In this condition the competition between the collisions experienced by the heavy disks from the caged light disks, which push them apart, and those experience from the surrounding disks, which push them toghether, is won by the caged disks and results in an effective repulsive force.

Segregation and instability - The effective force, $\mathbf{f}^{\mathrm{eff}}$, was derived for a system of just two heavy grains in a "bath" of lighter ones. For a system with many heavy grains this is, in general, expected to be just an approximation. We checked, however, that $\mathbf{f}^{\text {eff }}$ captures the basic physical mechanism responsible for segregation via stripes formation in the investigated system. To this aim we run simulations of a monodisperse system of heavy disks only, where disks interact pairwise via the effective force previously determined. As in the original mixture the disks are also subject to a viscous force $\left(\mathbf{f}_{\text {tray }}=-\mu_{\mathrm{h}} \mathbf{v}\right)$ and to a white noise force $\xi$. They are placed on a fixed tray of size $160 D \times 40 D$ and cover an area fraction $\phi \simeq 0.5$.

Fig. 3 shows that this system evolves, from an initially disordered configuration, via the formation of interconnected clusters that at long times break into a pattern of stripes parallel to the $y$ direction. This is precisely the behavior exhibited by each single component of the 

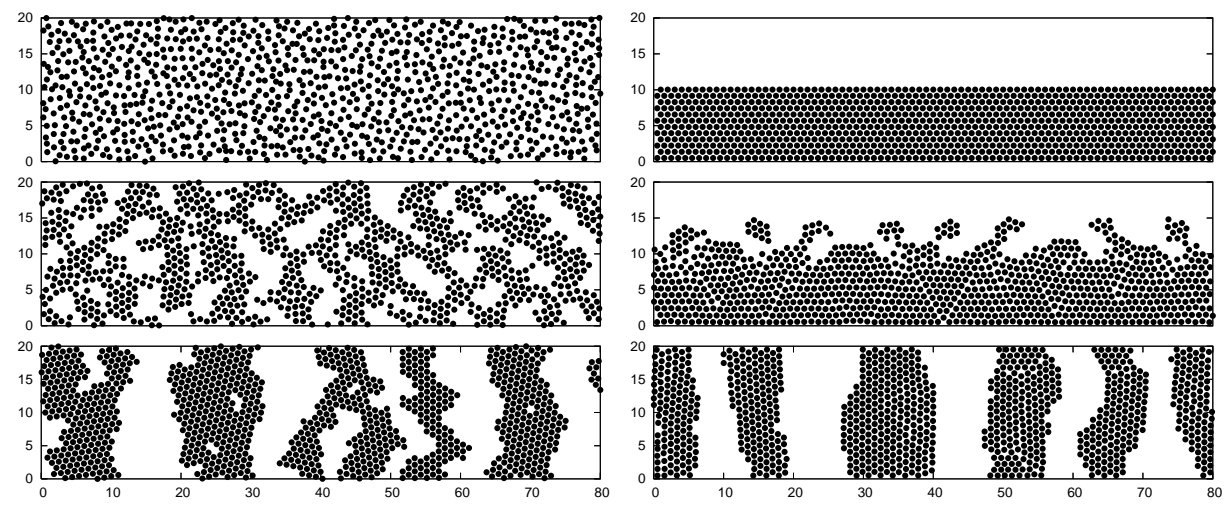

FIG. 3: Evolution of a system of disks interacting via the effective force shown in Fig. 11 Lenghts are expressed in units of particles diameters. Left column (periodic boundary conditions in both direction): from top to bottom, evolution from an intially disorder state at $t=0,4$ and 100s. Right column (periodic boundary condition along $x$, elastic along $y$ ): evolution from an initially segregated state at $t=0,8$ and 300s. Each single component of a real granular mixtures subject to horizontal oscillations evolves in a similar way [2].

original horizontally oscillated binary mixture of disks in simulations (see Fig.1b of Ref. [2]) and experiments [9]. An insight on the system behavior can be gained via an analogy with thermal systems with short range attraction and long range repulsion [14]. In these systems the short range attraction, which tends to induce a macroscopic phase separation with the formation of a single large cluster in the system, is frustrated by the presence of the long range repulsion adversing large clusters. Depending on the relative strength of attraction and repulsion, the attraction may dominates and the system will eventually phase separate via the standard coarsening mechanism, otherwise if the repulsion dominates the coarsening process eventually stops when a typical domain width is reached, resulting in the formation of striped patterns [14]. In our granular system, as the long range repulsive component of the effective force vanishes along the $y$ axis (see Fig. 1 $\theta=\pi / 2$ ), cluster growth along $y$ is not frustrated. Along $x$ the relative strength of the repulsion is small compared to the attraction, and the coarsening process will therefore proceeds slowly as long as the external driving keeps going. The asymmetry of the interaction force therefore explains the formation and the orientation of the striped pattern.

At low values of the strength of the white noise force, $\Gamma$, the stripes appears to be formed by disks in an ordered (crystal) state (see Fig. 31). At a higher value of $\Gamma$ the stripes appears fluid-like, while at stiller higher values the system does not segregate. The same behavior is observed in a real mixture of disks subject to horizontal oscillation both when $\Gamma$ is increased, and when the area fraction of one of the two components is decreased [2, 9].

The effective interaction explains as well the occurrence of the dynamical instability generating the above segregated pattern [2]. The instability is best visualized when the initial state of the system is not disordered, but disks interacting with the effective force are placed on the tray in a stripe parallel to the $x$ direction (see Fig. 31). In this condition the initially flat free surface develops a sine-like modulation which grows until it breaks giving rise to a pattern of alternating stripes perpendicular to $x$ direction. The same phenomenology is observed when the two components of the granular mixture are placed on the oscillating tray in two stripes parallel to the $x$ direction (see, for instance, Fig.1a of Ref. [2]).

Cahn-Hilliard approach - As the Cahn-Hilliard equation captures the general features of spinodal decomposition of thermal binary mixtures (see [15] for a review), regardless of the details of the interaction potential between the two components, we expect that a phenomenological Cahn-Hilliard equation may capture the properties of our granular system when coarsening is observed. Therefore we have investigated a Cahn-Hilliard equation for the density difference $c(\vec{r})=\rho_{1}(\vec{r})-\rho_{2}(\vec{r})$ of the two components, which takes into account the anisotropy found in the effective force field

$$
\frac{\partial c(\vec{r})}{\partial t}=M \nabla^{2}\left[\frac{\partial f}{\partial c}-\left(K_{x} \partial_{x}^{2}+K_{y} \partial_{y}^{2}\right) c\right]+\eta
$$

Here $M$ is a mobility and $\eta$ a Gaussian random noise [16], $f(c)$ the usual double-well potential leading to phase separation, whereas the term $K_{x} \partial_{x}^{2}+K_{y} \partial_{y}^{2}$ accounts for the "free energy" cost associated to concentration gradients. In the present case, as the effective interaction is not spherically invariant, the cost of an interface depends on its orientation. Schematically we take this into account by assuming $K_{y}>K_{x}$ (in case of radial isotropy $K_{x}=K_{y}$ ) since interfaces (i.e., concentration gradients) along $y$ "cost more" than interfaces along $x$. We show in

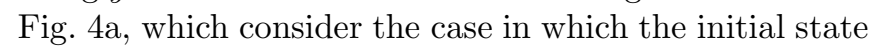
is homogeneous, and in Fig. $4 \mathrm{~b}$, which reproduces the instability of an initially flat interface between the two 
a

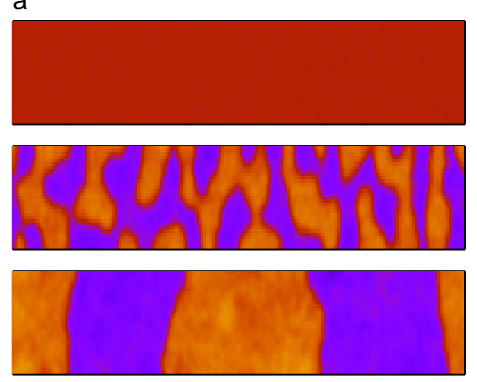

b

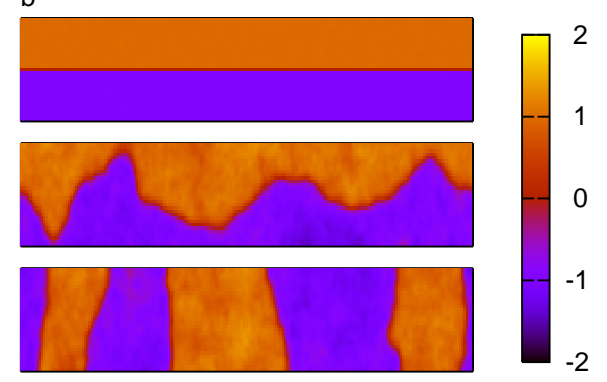

FIG. 4: (color on line) Solution of the modified Cahn-Hilliard equation (Eq. 2), with $K_{y} / 2=K_{x}=1$ in a system of size $200 \times 32$ with periodic boundary conditions along $x$ and no flux boundary conditions along $y$. (a): evolution from an homogeneous initial condition shown at times $t=0,100,2500$; (b) evolution from a segregated initial condition shown at times $t=0,750,2500$. The color scale on the right is a measure of the density difference field.

components, that this phenomenological equation gives a good description of the dynamics. A quantitative agrement is also found as the Cahn-Hilliard equation predicts a coarsening exponent $\nu=1 / 4$ during the first coarsening regime 17], which is numerically equal to the one observed in both experiments and simulations [7, 9]. According to the Cahn-Hilliard equation we expect, however, a crossover 17] to $\nu=1 / 3$ at much longer times, not yet observed in experiments and simulations.

Conclusions - In this Letter we have generalized the effective interaction approach, widely used in the study of colloidal systems, to out-of equilibirum periodically driven mixtures, and specifically to the case of a granular mixture subject to horizontal oscillations. In this context the effective interaction approach is particularly useful, as it reduces the study of an out-of equilibrium non-thermal driven system (dissipative in the case we have explicitely investigated), to that of an "equilibirum" monodisperse system. For a granular mixture subject to horizontal oscillations, the effective interaction force, whose foundamental chracteristics is its directional anisotropy, and particularly the presence of a repulsive shoulder at long distances which is prominent in the direction of oscillation, allows for a clear understanding of the observed instabilities and segregation processes. Its features can be casted in a phenomenological CahnHilliard equation which reproduces the observed phenomenology and allows for analytical predictions. Our findings clarify, thus, the origin of the "differential drag" mechanism [11] proposed to describe the observed phenomena and show how to interpret phenomenological hydrodynamics models [10] used to depict the early stages of the stripe dynamics.

Work supported by EU Network Number MRTN-CT2003-504712, MIUR-PRIN 2004, CrdC-AMRA.

\footnotetext{
picaciamarra@na.infn.it
}

† URL: http://smcs.na.infn.it

[1] D.J. Goldfarb, B.J. Glasser and T. Shinbrot, Nature 415, 302 (2002).

[2] M. Pica Ciamarra, M. Nicodemi and A. Coniglio, Phys. Rev. Lett. 94, 188001 (2005).

[3] T. Shinbrot and F.J. Muzzio, Nature 410, 251 (2001).

[4] P. Richard et al., Nature Materials 4, 121 (2005).

[5] J.M. Ottino and D.V. Khakhar, Ann. Rev. Fluid Mech. 32, 55 (2000); A. Kudrolli, Rep. Prog. Phys. 67, 209 (2004).

[6] M. Pica Ciamarra et. al., Phys. Rev. Lett. 96, 058001 (2006).

[7] M. Pica Ciamarra, M. Nicodemi and A. Coniglio, J. Phys.: Condens. Matter 17, S2549 (2005); M. Pica Ciamarra, A. Sarracino, M. Nicodemi and A. Coniglio, in preparation.

[8] S. Asakura and F. Oosawa, J. of Poly. Sci. 33, 183 (1958).

[9] T. Mullin, Phys. Rev. Lett. 84, 4741 (2000); P.M. Reis and T. Mullin, Phys. Rev. Lett. 89, 244301 (2002) P.M. Reis et al., Europhys. Lett. 66, 357 (2004).

[10] C.M. Pooley and J.M. Yeomans, Phys. Rev. Lett. 93, 118001 (2004).

[11] P. Sánchez, M.R. Swift and P.J. King, Phys. Rev. Lett. 93184302 (2004).

[12] L.E. Silbert et al., Phys Rev E 64, 051302 (2001).

[13] J. Dzubiella, H. Lowen and C.N. Likos, Phys. Rev. Lett. 91, 248301 (2003).

[14] S. Glotzer and A. Coniglio, Phys. Rev. 50, 4241 (1994); M. Seul and D. Andelman, Science 267, 476 (1995); A. Imperio and L. Reatto, Phys.: Condens. Matter 16, S3769 (2004).

[15] N. Goldenfeld, Lectures on phase transitions and the renormalization group, Addison-Wesley, 1992; A. Bray, Adv. Phys. 43, 357 (1994).

[16] The Gaussian random noise $\eta$ is obtained as the divergence of a random vector field in order to keep the order parameter conserved.

[17] F. Corberi, A. Coniglio and M. Zannetti, Phys. Rev. E 51, 5469 (1995); C. Castellano and M. Zannetti, Phys. Rev. E 58, 5410 (1998).

[18] According to Helmholtz's theoreom any vector field $\mathbf{V}$ admits a unique decomposition in a irrotational and solenoidal component, $\mathbf{V}=\mathbf{V}_{I}+\mathbf{V}_{S}$. The solenoidal component of $\mathbf{f}^{\text {eff }}$ appears to be negligible with respect to its irrotatioanl component, $\mathbf{f}_{S}(r) \ll \mathbf{f}_{I}(r)$. 